\title{
Proactive Hedging European Option Pricing with a General Logarithmic Position Strategy
}

\author{
Lixin Qiao, ${ }^{1}$ Fangfang Sun, ${ }^{1}$ Xiaotuo Qiao, ${ }^{2}$ Meng Li $\mathbb{D},{ }^{1}$ and Xuefeng Wang ${ }^{1}$ \\ ${ }^{1}$ School of Management, Harbin Institute of Technology, Harbin 150001, China \\ ${ }^{2}$ School of Finance, Zhongnan University of Economics and Law, Wuhan, China \\ Correspondence should be addressed to Meng Li; rilanhio@hit.edu.cn
}

Received 15 October 2021; Revised 15 December 2021; Accepted 18 December 2021; Published 19 January 2022

Academic Editor: Sundarapandian Vaidyanathan

Copyright (c) 2022 Lixin Qiao et al. This is an open access article distributed under the Creative Commons Attribution License, which permits unrestricted use, distribution, and reproduction in any medium, provided the original work is properly cited.

\begin{abstract}
This study proposes an exotic option that extends the classical European option by requiring option holders to continuously trade in underlying assets according to a predesignated trading strategy with a general logarithmic position. The pricing formula for the exotic option with a general logarithmic strategy is derived from the Black-Scholes option pricing formula, and its price advantage is compared (based on simulations) to the classical European option and to the exotic option with a linear position. By varying key parameters, we found that the exotic option with a general logarithmic position has a significant price advantage (up to $34 \%$ under certain parameter settings) over the classical European option. Moreover, the exotic option with a general logarithmic strategy can save $5.5 \%$ more of the option premium than applying a linear position strategy. Our simulation results indicate that the price advantage of this proactive hedging option with a general logarithmic strategy depends heavily on the initial amount of capital; in particular, this exotic option is more suitable for traders with limited initial amounts of capital.
\end{abstract}

\section{Introduction}

Options comprise a diverse group of indispensable trading products in the financial market, which make them effective tools for hedging risks. The options currently being traded include more than the standardized European and American options; in fact, a vast number of exotic options (e.g., barrier options, Asian options, lookback options, step options, rainbow options, binary options, and basket options, among others) are being changed, combined, and derived from the standard options [1-12]. These exotic options provide traders with more choices for outlining their trading portfolio strategies. In particular, they have the opportunity to finely match the nuances of traders' market expectations and to satisfy their risk-return needs under various market conditions.

In contrast to other financial instruments, the price of an option is not the value of the underlying asset, but rather, it is based on the hedging cost. In particular, the cheaper the option, the more favorable its price discovery. In addition, a portfolio strategy with cheaper options is easier to execute because the option price determines the break-even threshold. However, few studies have evaluated the price advantages of various options. Therefore, one of the main aims of this report is to propose an exotic option that can satisfy traders' needs for a cheaper price.

The pricing of options depends on the risk exposure of their underlying assets. The greater the risk exposure of the underlying asset, the more expensive the option. On this basis, Wang et al. proposed an exotic option that requires option holders to buy-in (or sell out) the underlying asset (stock) and allows them to adjust the holdings of the underlying asset according to its price changes within an option period [13-15]; this option was later named the proactive hedging European option by Li et al. [16]. For a given call option, the option buyer initially holds a certain amount of capital $(A)$. When the price of the underlying assets exceeds a designated threshold (usually the exercise price), the option contract requires the option holder to continuously buy the underlying asset following the preagreed dynamic investment strategy. If the option is exercised, the seller of the call option only needs to bear the expected loss corresponding to the difference between the purchase price and the exercise price. Therefore, the pricing of this exotic option depends on 
the amount of residual exposure of the aforementioned dynamic position strategy. Simulations have indicated that the price of a proactive hedging European option is significantly lower than that of the analogous classical option. However, Wang et al. assumed that the dynamic position strategy was a continuous linear function, and this assumption is not perfect. For example, the trading behavior of traders is discontinuous, and therefore, it does not always satisfy the assumed continuous function. Li et al. improved the continuous linear function to establish a discrete linear dynamic position strategy [17], although a nonlinear dynamic position strategy might be more suitable for obtaining cheaper options. In an extreme case, if a buyer is purchasing the underlying asset with all of the capital at one time at the exercise price, then the risk exposure of the underlying asset would be completely covered, and the theoretical price of the option will be zero. It is, therefore, clear that for European call options, the greater the proportion of purchasing that occurs near the threshold (i.e., the exercise price), the greater amount of exposure that is covered. Therefore, a nonlinear dynamic position strategy should be considered.

This study improves the linear position strategy by developing a nonlinear function and subjectively setting it as a general logarithmic function. The theoretical price of the option is derived, and its price advantage is evaluated. The remainder of this report is organized as follows. Section 2 describes the exotic option with a general logarithmic position strategy in detail and derives its value function; Section 3 obtains the theoretical price; Section 4 discusses two special cases of the exotic option; and Section 5 compares the theoretical prices of the exotic options with those of the classical European options based on simulations. Since the derivation processes are very similar for call and put options, we only present the results for call options herein.

\section{Proactive Hedging European Option with a General Logarithmic Position Strategy}

2.1. Assumptions for the Proactive Hedging European Option. The proactive hedging European option is proposed based on the following assumptions:
(1) A call option holder should hold initial capital in the amount of $A=Q \times X_{e}$ at the beginning of the option period for each part of the option contract, where $Q$ is the underlying asset amount of one part of the option contract, and $X_{e}$ is the exercise price.

(2) Option holders adjust the underlying asset holdings according to the price changes, which are subject to the general logarithmic position constraints of the option contract. The detailed dynamic position strategy will be introduced in Section 2.2.

(3) The price of the underlying asset follows geometric Brownian motion (GBM).

(4) There are no other cash flows for the underlying assets during the option contract period. For example, stocks do not pay dividends.

(5) There are no transaction costs for buying or selling the underlying assets.

(6) The risk-free interest rate is constant over time.

2.2. General Logarithmic Position Strategy. When the underlying asset price reaches $X_{e}+\delta$ (where $\delta \geq 0$ ), the general logarithmic position strategy is activated. Then, the option holder spends $\beta_{0} \cdot A$ to buy the underlying asset, where $\beta_{0} 0 \leq \beta_{0} \leq 1$ is the "initial capital utilization coefficient," which denotes the proportion of capital spent.

As the underlying asset price rises, the option holder will continuously increase their capital utilization, i.e., by buying the underlying asset in accordance with the general logarithmic function, $\beta_{0} \cdot A$, where $\beta 0 \leq \beta_{0} \leq \beta \leq 1$ is the "highest capital utilization coefficient." At this time, the underlying asset price reaches $(1+\alpha)\left(X_{e}+\delta\right)$, where $\alpha$ is the "investment strategy index," which is a positive number. The greater the value of $\alpha$, the larger the price range where traders can buy the underlying asset. The value of $\alpha$ is set within the range $[0.5,1]$ to encompass the various risk preferences of traders. Figure 1 illustrates the described process.

The capital utilization coefficient function associated with this process, $B(S)$, can be expressed as follows:

$$
B(S)= \begin{cases}0, & S<X_{e}+\delta, \\ \beta_{0}+\frac{\beta-\beta_{0}}{\ln (1+\alpha)} \ln \left(\frac{S}{X_{e}+\delta}\right), & X_{e}+\delta \leq S<(1+\alpha)\left(X_{e}+\delta\right), \\ \beta, & (1+\alpha)\left(X_{e}+\delta\right) \leq S .\end{cases}
$$

contract as the underlying asset prices rise from $X_{e}$ to $S_{0}$, for $S_{0}>X_{e}$.

$$
L_{b}\left(S_{0}\right)=Q\left(S_{0}-X_{e}\right)=\frac{A}{X_{e}}\left(S_{0}-X_{e}\right) .
$$

2.3. The Value Function for a Proactive Hedging European Option with a General Logarithmic Position Strategy. For the classical European option without a proactive hedging strategy, the option holder would suffer an expected loss, $L$, according to equation (2), for each part of the option 


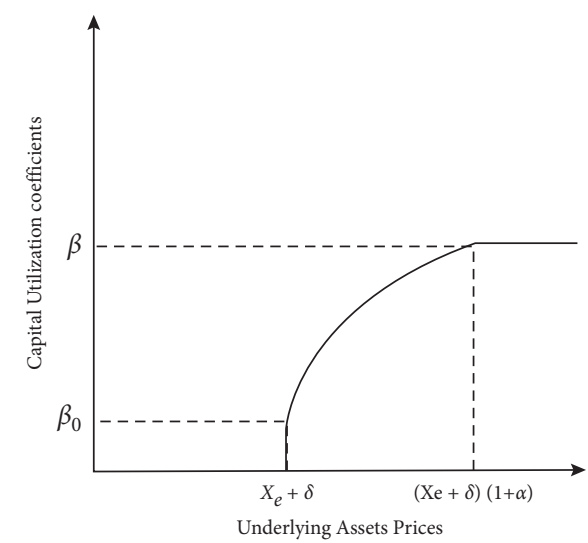

FIGURE 1: Graphical illustration of the general logarithmic position strategy.

In an exotic option with a proactive hedging strategy, the option holder is required to actively buy-in the underlying assets to hedge the risks. If we assume that the option holder trades using the general logarithmic position strategy described in Section 2.2 and buys the underlying asset of the contract piece $A \cdot \beta_{0} / X_{e}+\delta$ when the underlying asset price
$S$ reaches $X_{e}+\delta$, then, when the underlying asset price $S$ changes to $S+\Delta S$, the option holder increases their capital by $\Delta q$ to buy shares, where $\Delta q=\Delta B \Delta A=A \Delta B^{\prime}(S) \Delta \Delta S$. Furthermore, when the underlying asset price $S$ rises from $X_{e}+$ $\delta$ to $S_{0}$, the expected return obtained by the option holder is given by

$$
R\left(S_{0}\right)= \begin{cases}0, & S(t)<X_{e}+\delta, \\ \int_{X_{e}+\delta}^{S_{0}} \frac{A \cdot B^{\prime}(S)}{S}\left(S_{0}-S\right) \mathrm{d} S+\frac{A \cdot \beta_{0}}{X_{e}+\delta}\left(S_{0}-X_{e}-\delta\right), & X_{e}+\delta \leq S_{0}<(1+\alpha)\left(X_{e}+\delta\right), \\ \int_{X_{e}+\delta}^{(1+\alpha)\left(X_{e}+\delta\right)} \frac{\mathrm{A} \cdot B^{\prime}(S)}{S}\left(S_{0}-S\right) \mathrm{d} S+\frac{A \cdot \beta_{0}}{X_{e}+\delta}\left(S_{0}-X_{e}-\delta\right), & S_{0} \geq(1+\alpha)\left(X_{e}+\delta\right) .\end{cases}
$$

Substituting equation (1) into equation (3) gives

$$
R\left(S_{0}\right)= \begin{cases}0, & S(t)<X_{e}+\delta, \\ \frac{A\left(\beta-\beta_{0}\right)}{\ln (1+\alpha)}\left[\frac{S_{0}}{X_{e}+\delta}-\ln \left(\frac{S_{0}}{X_{e}+\delta}\right)-1\right]+\frac{A \cdot \beta_{0}}{X_{e}+\delta}\left(S_{0}-X_{e}-\delta\right), & X_{e}+\delta \leq S_{0}<(1+\alpha)\left(X_{e}+\delta\right), \\ \frac{A \cdot \alpha \cdot\left(\beta-\beta_{0}\right) \cdot S_{0}}{\left(X_{e}+\delta\right)(1+\alpha) \ln ((1+\alpha))}-A \cdot\left(\beta-\beta_{0}\right)+\frac{A \cdot \beta_{0}}{X_{e}+\delta}\left(S_{0}-X_{e}-\delta\right), & S_{0} \geq(1+\alpha)\left(X_{e}+\delta\right) .\end{cases}
$$

If the expected loss born by the option seller is defined by

$$
L\left(S_{0}\right)=L_{b}\left(S_{0}\right)-R\left(S_{0}\right) \text {, }
$$

then 


$$
L\left(S_{0}\right)= \begin{cases}\frac{A}{X_{e}}\left(S_{0}-X_{e}\right), & S_{0}<X_{e}+\delta, \\ \frac{A}{X_{e}}\left(S_{0}-X_{e}\right)-\left\{\frac{A \cdot\left(\beta-\beta_{0}\right)}{\ln (1+\alpha)}\left[\frac{S_{0}}{X_{e}+\delta}-\ln \left(\frac{S_{0}}{X_{e}+\delta}\right)-1\right]+\frac{A \cdot \beta_{0}}{X_{e}+\delta}\left(S_{0}-X_{e}-\delta\right)\right\}, & X_{e}+\delta \leq S_{0}<(1+\alpha)\left(X_{e}+\delta\right), \\ \frac{A}{X_{e}}\left(S_{0}-X_{e}\right)-\left[\frac{A \cdot \alpha \cdot\left(\beta-\beta_{0}\right) \cdot S_{0}}{\left(X_{e}+\delta\right) \cdot(1+\alpha) \cdot \ln (1+\alpha)}-A\left(\beta-\beta_{0}\right)+\frac{A \cdot \beta_{0}}{X_{e}+\delta}\left(S_{0}-X_{e}-\delta\right)\right], & S_{0} \geq(1+\alpha)\left(X_{e}+\delta\right),\end{cases}
$$

Thus, the option contract value $V_{T}(S(t), t)$ corresponding to a unit of the underlying asset is given by

$$
V_{T}(S(t), t)=\frac{L(S(t))}{\left(A / X_{e}\right)}= \begin{cases}V_{T 1}(S(t), t), & S(t)<X_{e}, \\ V_{T 2}(S(t), t), & X_{e} \leq S(t)<X_{e}+\delta, \\ V_{T 3}(S(t), t), & X_{e}+\delta \leq S(t)<(1+\alpha)\left(X_{e}+\delta\right), \\ V_{T 4}(S(t), t), & S(t) \geq(1+\alpha)\left(X_{e}+\delta\right),\end{cases}
$$

where

$$
\begin{aligned}
& V_{T 1}(S(t), t)=0, \\
& V_{T 2}(S(t), t)=S(t)-X_{e}, \\
& V_{T 3}(S(t), t)=\left(S(t)-X_{e}\right)\left(1-\frac{\beta_{0} \cdot X_{e}}{X_{e}+\delta}\right)-\frac{\left(\beta-\beta_{0}\right) \cdot X_{e}}{\ln (1+\alpha)}\left[\frac{S(t)}{X_{e}+\delta}-\ln \left(\frac{S(t)}{X_{e}+\delta}\right)\right]+\frac{\left(\beta-\beta_{0}\right) \cdot X_{e}}{\ln (1+\alpha)}+\frac{\beta_{0} \cdot X_{e} \cdot \delta}{X_{e}+\delta}, \\
& V_{T 4}(S(t), t)=\left(S(t)-X_{e}\right)\left(1-\frac{\beta_{0} \cdot X_{e}}{X_{e}+\delta}\right)-\frac{\alpha \cdot\left(\beta-\beta_{0}\right) \cdot X_{e} \cdot S(t)}{\left(X_{e}+\delta\right) \cdot(1+\alpha) \cdot \ln (1+\alpha)}+X_{e} \cdot\left(\beta-\beta_{0}\right)+\frac{\beta_{0} \cdot X_{e} \cdot \delta}{X_{e}+\delta} .
\end{aligned}
$$

Corollary 1. The proactive hedging European option with a general logarithmic position strategy will cover more risk exposure of the underlying assets than the same option with a general linear position strategy.

Proof. To simplify the proof process, the dynamic position strategy was reduced to the case where $\delta=0$ and $\beta_{0}=0$.
Then, the capital utilization coefficient function of the general logarithmic position strategy and the linear position strategy is denoted as $B_{\mathrm{LG}}(S)$ and $B_{\mathrm{LN}}(S)$, which is expressed by equations (9) and (10), respectively, and depicted in Figure 2:

$$
\begin{aligned}
& B_{\mathrm{LG}}(S)= \begin{cases}0, & S(t)<X_{e}, \\
\frac{\beta}{\ln (1+\alpha)} \ln \left(\frac{S(t)}{X_{e}}\right), & X_{e} \leq S(t)<X_{e}(1+\alpha), \\
\beta, & X_{e}(1+\alpha) \leq S(t),\end{cases} \\
& B_{\mathrm{LN}}(S)= \begin{cases}0, & S(t)<X_{e}, \\
\frac{\beta}{\alpha \cdot X_{e}}\left(S(t)-X_{e}\right), & X_{e} \leq S(t)<X_{e}(1+\alpha), \\
\beta, & X_{e}(1+\alpha) \leq S(t) .\end{cases}
\end{aligned}
$$




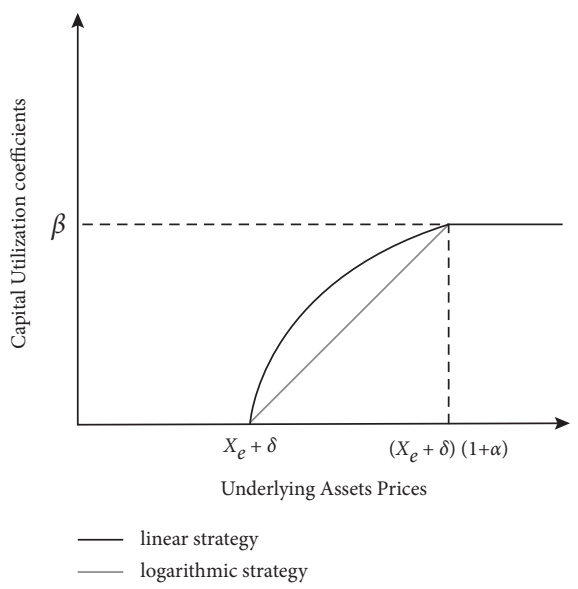

FIgURE 2: Capital utilization coefficients of the logarithmic strategy vs. the linear strategy.

In the same way, the expected returns of the general logarithmic and linear position strategies can be obtained based on $R_{\mathrm{LG}}(S(t))$ and $R_{\mathrm{LN}}(S(t))$, respectively, where $R_{\mathrm{LG}}(S(t))$ (equation (11)) is derived from the special case where $\delta=0$ and $\beta_{0}=0$ equation (3), and $R_{\mathrm{LG}}(S(t))$ equation (12) is derived from the special case where $\delta=0$ and $\beta_{0}=0$, as in the study conducted by Wang et al. [13].

$$
\begin{aligned}
& R_{\mathrm{LG}}(S(t))= \begin{cases}0, & S(t)<X_{e}, \\
\frac{A \cdot \beta}{\ln (1+\alpha)}\left[\frac{S(t)}{X_{e}}-\ln \left(\frac{S(t)}{X_{e}}\right)-1\right], & X_{e} \leq S(t)<(1+\alpha) X_{e}, \\
A \cdot \beta\left[\frac{\alpha \cdot S(t)}{X_{e}(1+\alpha) \ln (1+\alpha)}-1\right], & S(t) \geq(1+\alpha) X_{e},\end{cases} \\
& R_{\mathrm{LN}}(S(t))= \begin{cases}0, & S(t)<X_{e}, \\
A \cdot \beta\left[\frac{S(t)}{\alpha \cdot X_{e}} \ln \left(\frac{S(t)}{X_{e}}\right)-\frac{S(t)-X_{e}}{\alpha \cdot X_{e}}\right], & X_{e} \leq S(t)<(1+\alpha) X_{e}, \\
A \cdot \beta\left[\frac{S(t)}{\alpha \cdot X_{e}} \ln (1+\alpha)-1\right], & S(t) \geq(1+\alpha) X_{e} .\end{cases} \\
& \begin{array}{ccc}
\alpha^{2}-(1+\alpha) \ln ^{2}(1+\alpha) \geq 0, & f_{\max }^{\prime}(x)=f^{\prime}(2)=3-(\ln 2+1)^{2}>0, \\
\text { that } R_{\mathrm{LG}}(S(t)) \geq R_{\mathrm{LN}}(S(t)) \quad \text { when } & (13) & f_{\min }^{\prime}(x)=f^{\prime}\left(\frac{3}{2}\right)=2-\left(\ln \frac{3}{2}+1\right)^{2}>0 .
\end{array}
\end{aligned}
$$

$S(t) \geq(1+\alpha) X_{e}$, the following inequality should be satisfied:

where $\alpha \in[0.5,1]$ (according to the aforementioned definition of the general logarithmic position strategy) is always true. The inequality in equation (13) is proved as follows.

Set $x=1+\alpha$, then $x \in[3 / 2,2]$, and inequality (13) can be rewritten as $(x-1)^{2}-x \ln ^{2} x>0$. Set $f(x)=(x-1)^{2}$ $-x \ln ^{2} x$, then $f^{\prime}(x)=2 x-2-2 \ln x-\ln ^{2} x$, and $f^{\prime \prime}(x)=2 x-2-2 \ln x / x$.

It is known that under the condition $x \in[3 / 2,2]$, we can define $x-1>\ln x$, and $f^{\prime \prime}(x)>0$ holds on [3/2,2], while $f^{\prime}(x)$ is a monotonically increasing function.

Therefore, and $f^{\prime}(x)>0$ is always true on $[3 / 2,2]$, while $f(x)>0$ monotonically increases on $[3 / 2,2]$.

As a result,

$$
f_{\min }(x)=f\left(\frac{3}{2}\right)=\frac{1}{4}-\frac{3}{2} \ln ^{2} \frac{3}{2}>0,
$$

is sufficient.

When $X_{e} \leq S(t)<(1+\alpha) X_{e}$, a similar procedure can be applied; therefore, a detailed verification process is omitted here. 


\section{Proactive Hedging Option Pricing Formula Based on GBM}

3.1. Asset Price Behavior Based on GBM. Let $S(t)$ be the asset price at the time $t$. If $S(t)$ follows GBM, then it satisfies the following equation:

$$
\frac{\mathrm{d} S(t)}{S(t)}=\mu \mathrm{d} t+\sigma \mathrm{d} B_{t}
$$

where $S(0)$, the draft $\mu$, and the volatility $\sigma$ of the asset price are all positive constants for $t \geq 0$.

By applying the fractional Wick-Itô formula, $\mathrm{Hu}$ and Øksendal [18] proved that equation (16) can be rewritten as follows:

$$
S(t)=S(0) \exp \left(\sigma B(t)+\mu t-\frac{1}{2} \sigma^{2} t\right)
$$

Assuming risk-neutral conditions, i.e., $\mu=r$, then, for any two time points $t_{1}$ and $t_{2}$ where $0 \leq t_{1} \leq t_{2} \leq T$, the relationship between $S\left(t_{1}\right)$ and $S\left(t_{2}\right)$ can be obtained from equation (17) such that

$S\left(t_{2}\right)=S\left(t_{1}\right) \exp \left[r\left(t_{2}-t_{1}\right)-\frac{1}{2} \sigma^{2}\left(t_{2}-t_{1}\right)+\sigma\left(B\left(t_{2}\right)-B\left(t_{1}\right)\right)\right]$.
3.2. European Option Pricing Formula. Black and Scholes (1973) derived the famous B-S partial differential function for determining the theoretical price of a classical European option by applying Ito's Lemma, as follows:

$$
\left\{\begin{array}{l}
\frac{\partial V}{\partial t}+r S \frac{\partial V}{\partial S}+\frac{1}{2} \sigma^{2} S^{2} \frac{\partial^{2} V}{\partial S^{2}}=r f, \\
V(S, T)=f(S, T),
\end{array}\right.
$$

where $f$ is the option price, $t$ is the time, $r$ is the risk-free return rate, $\sigma$ is the volatility of the stock return, and $S$ is the stock price.

Theorem 1 (also Theorem 1 in ref. [19]). Let $f$ be $a$ function, such that $E\left(f\left(B_{t}\right)\right)<\infty$. Then, for every $0 \leq t \leq T$,

$\widehat{E}\left(f\left(B_{t}\right)\right)=\int_{-\infty}^{+\infty} \frac{1}{\sqrt{2 \pi(T-t)}} \exp \left(-\frac{\left(x-B_{t}\right)^{2}}{2(T-t)}\right) \cdot f(x) \mathrm{d} x$.

Proposition 1. The analytical solution to the B-S formula (19) under GBM is given by.

$$
V(S(t), t)=\frac{e^{-r(T-t)}}{\sqrt{2 \pi}} \int_{-\infty}^{+\infty} f\left(S(t) e^{\sigma \sqrt{T-t} \cdot Z+(T-t)\left(r-\sigma^{2} / 2\right)}\right) \cdot e^{-\left(Z^{2} / 2\right)} \mathrm{d} Z
$$

where $T$ is the option period, and $f(\cdot)$ is the intrinsic value function of the option. The intrinsic value of an option is the value of the option at a maturity date. $t=T$.

Proof. According to the Feynman-Kac formula, we can define the function as follows:

$$
V(S, t)=E^{S}\left[e^{-\int_{t}^{T} r \mathrm{~d} t} f(S(T))\right]
$$

Then, the $V(S, T)$ satisfies the following partial differential equation:

$$
\begin{aligned}
V(S, t) & =e^{-r(T-t)} E^{S}[f(S(T))] \\
& =e^{-r(T-t)} \widehat{E}\left[f\left(S \cdot \exp \left[r(T-t)-\frac{1}{2} \sigma^{2}(T-t)+\sigma\left(B_{T}-B_{t}\right)\right)\right]\right. \\
& =\frac{e^{-r(T-t)}}{\sqrt{2 \pi(T-t)}} \int_{-\infty}^{+\infty} \exp \left(-\frac{\left(x-B_{t}\right)^{2}}{2(T-t)}\right) f\left(S \cdot \exp \left[r(T-t)-\frac{1}{2} \sigma^{2}(T-t)+\sigma\left(x-B_{t}\right)\right]\right) \mathrm{d} x
\end{aligned}
$$

By setting $Z=\left(x-B_{t}\right) / \sqrt{(T-t)}$, we can obtain the integral equation (20), and the proof is complete.

$$
\frac{\partial V}{\partial t}+r S \frac{\partial V}{\partial S}+\frac{1}{2} \sigma^{2} S^{2} \frac{\partial^{2} V}{\partial S^{2}}=r f
$$

with the terminal condition:

$$
V(S, T)=f(S(T))=\max (S(T)-K, 0) .
$$

To obtain the analytical solution of the option pricing formula in equation (22) under the GBM assumption, we apply Theorem 1 and combine equations (18) and (22), such that 
is a stepwise function, and therefore, the pricing formula where $V_{C}(S(t), t)$ includes four components, as follows:

$$
\begin{aligned}
V_{C}(S(t), t)= & V_{1}(S(t), t)+V_{2}(S(t), t) \\
& +V_{3}(S(t), t)+V_{4}(S(t), t),
\end{aligned}
$$

$$
\begin{aligned}
& V_{1}(S(t), t)=\frac{e^{-r(T-t)}}{\sqrt{2 \pi}} \int_{S<X_{e}} V_{T 1}\left(S(t) \cdot e^{\sigma \sqrt{T-t} \cdot Z+(T-t)\left(r-\left(\sigma^{2} / 2\right)\right)}, t\right) e^{-\left(Z^{2} / 2\right)} \mathrm{d} Z, \\
& V_{2}(S(t), t)=\frac{e^{-r(T-t)}}{\sqrt{2 \pi}} \int_{X_{e} \leq S<X_{e}+\delta} V_{T 2}\left(S(t) \cdot e^{\sigma \sqrt{T-t} \cdot Z+(T-t)\left(r-\left(\sigma^{2} / 2\right)\right)}, t\right) e^{-\left(Z^{2} / 2\right)} \mathrm{d} Z, \\
& V_{3}(S(t), t)=\frac{e^{-r(T-t)}}{\sqrt{2 \pi}} \int_{X_{e}+\delta \leq S<(1+\alpha)}\left(X_{e}+\delta\right) \\
& V_{T 3}\left(S(t) \cdot e^{\sigma \sqrt{T-t} \cdot Z+(T-t)\left(r-\left(\sigma^{2} / 2\right)\right)}, t\right) e^{-\left(Z^{2} / 2\right)} \mathrm{d} Z, \\
& V_{4}(S(t), t)=\frac{e^{-r(T-t)}}{\sqrt{2 \pi}} \int_{S \geq(1+\alpha)\left(X_{e}+\delta\right)} V_{T 4}\left(S(t) \cdot e^{\sigma \sqrt{T-t} \cdot Z+(T-t)\left(r-\left(\sigma^{2} / 2\right)\right)}, t\right) e^{-\left(Z^{2} / 2\right)} \mathrm{d} Z .
\end{aligned}
$$

Therefore, $V_{1}(S(t), t)=0$.

For simplicity of $U=S \cdot e^{\sigma \sqrt{T-t} \cdot Z+(T-t)\left(r-\left(\sigma^{2} / 2\right)\right)}$.

identification, let

When $X_{e} \leq U<X_{e}+\delta$, then

$$
\frac{\ln X_{e} / S-(T-t)\left(r-\sigma^{2} / 2\right)}{\sigma \sqrt{T-t}} \leq Z<\frac{\ln X_{e}+\delta / S-(T-t)\left(r-\sigma^{2} / 2\right)}{\sigma \sqrt{T-t}} .
$$

When $X_{e}+\delta \leq U<(1+\alpha)\left(X_{e}+\delta\right)$, then

$$
\frac{\ln X_{e}+\delta / S-(T-t)\left(r-\sigma^{2} / 2\right)}{\sigma \sqrt{T-t}} \leq Z<\frac{\ln (1+\alpha)\left(X_{e}+\delta\right) / S-(T-t)\left(r-\sigma^{2} / 2\right)}{\sigma \sqrt{T-t}}
$$

When $U \geq(1+\alpha)\left(X_{e}+\delta\right)$, then

$$
Z \geq \frac{\ln (1+\alpha)\left(X_{e}+\delta\right) / S-(T-t)\left(r-\sigma^{2} / 2\right)}{\sigma \sqrt{T-t}} .
$$

To simplify these expressions, let 


$$
\begin{aligned}
& d_{1}=\frac{\ln X_{e} / S-(T-t)\left(r-\sigma^{2} / 2\right)}{\sigma \sqrt{T-t}}, \\
& d_{2}=\frac{\ln X_{e}+\delta / S-(T-t)\left(r-\sigma^{2} / 2\right)}{\sigma \sqrt{T-t}}, \\
& d_{3}=\frac{\ln (1+\alpha)\left(X_{e}+\delta\right) / S-(T-t)\left(r-\sigma^{2} / 2\right)}{\sigma \sqrt{T-t}}, \\
& d_{4}=d_{1}-\sigma \sqrt{T-t}=\frac{\ln X_{e} / S-(T-t)\left(r+\sigma^{2} / 2\right)}{\sigma \sqrt{T-t}}, \\
& d_{5}=d_{2}-\sigma \sqrt{T-t}=\frac{\ln X_{e}+\delta / S-(T-t)\left(r+\sigma^{2} / 2\right)}{\sigma \sqrt{T-t}}, \\
& d_{6}=d_{3}-\sigma \sqrt{T-t}=\frac{\ln (1+\alpha)\left(X_{e}+\delta\right) / S-(T-t)\left(r+\sigma^{2} / 2\right)}{\sigma \sqrt{T-t}} .
\end{aligned}
$$

Then,

$$
\begin{aligned}
& V_{2}(S(t), t)=\frac{e^{-r(T-t)}}{\sqrt{2 \pi}} \int_{X_{e} \leq S<X_{e}+\delta}\left(S(t) \cdot e^{\sigma \sqrt{T-t} \cdot Z+(T-t)\left(r-\left(\sigma^{2} / 2\right)\right)}-X_{e}\right) e^{-\left(Z^{2} / 2\right)} \mathrm{d} Z \\
& =\frac{e^{-r(T-t)}}{\sqrt{2 \pi}} \int_{d_{1}}^{d_{2}}\left(S(t) \cdot e^{\sigma \sqrt{T-t} \cdot Z+(T-t)\left(r-\left(\sigma^{2} / 2\right)\right)}\right) \cdot e^{-\left(Z^{2} / 2\right)} \mathrm{d} Z-\frac{X_{e} \cdot e^{-r(T-t)}}{\sqrt{2 \pi}} \int_{d_{1}}^{d_{2}} e^{-\left(Z^{2} / 2\right)} \mathrm{d} Z \\
& =\frac{S(t)}{\sqrt{2 \pi}} \int_{d_{1}}^{d_{2}} e^{\sigma \sqrt{T-t} \cdot Z+(T-t)\left(r-\left(\sigma^{2} / 2\right)\right)-r(T-t)-\left(Z^{2} / 2\right)} \mathrm{d} Z-\frac{X_{e} \cdot e^{-r(T-t)}}{\sqrt{2 \pi}} \int_{d_{1}}^{d_{2}} e^{-\left(Z^{2} / 2\right)} \mathrm{d} Z \\
& =\frac{S(t)}{\sqrt{2 \pi}} \int_{d_{1}}^{d_{2}} e^{-(1 / 2)\left(Z^{2}-2 \sigma \sqrt{T-t} \cdot Z+\sigma^{2}(T-t)\right)} \mathrm{d} Z-\frac{X_{e} \cdot e^{-r(T-t)}}{\sqrt{2 \pi}} \int_{d_{1}}^{d_{2}} e^{-\left(Z^{2} / 2\right)} \mathrm{d} Z \\
& =\frac{S(t)}{\sqrt{2 \pi}} \int_{d_{1}}^{d_{2}} e^{-(1 / 2)(Z-\sigma \sqrt{T-t})^{2}} \mathrm{~d} Z-\frac{X_{e} \cdot e^{-r(T-t)}}{\sqrt{2 \pi}} \int_{d_{1}}^{d_{2}} e^{-\left(Z^{2} / 2\right)} \mathrm{d} Z \\
& =\frac{S(t)}{\sqrt{2 \pi}} \int_{d_{4}}^{d_{5}} e^{-(1 / 2)(Z-\sigma \sqrt{T-t})^{2}} d(Z-\sigma \sqrt{T-t})-X_{e} \cdot e^{-r(T-t)}\left(N\left(d_{2}\right)-N\left(d_{1}\right)\right) \\
& =S(t) \cdot\left(N\left(d_{5}\right)-N\left(d_{4}\right)\right)-X_{e} \cdot e^{-r(T-t)}\left(N\left(d_{2}\right)-N\left(d_{1}\right)\right) \text {. }
\end{aligned}
$$

The expression of $V_{T 3}(\cdot)$ must be simplified for the sake of feasibility; therefore, let

$$
\Sigma=\sigma \sqrt{T-t} \cdot Z+(T-t)\left(r-\frac{\sigma^{2}}{2}\right) .
$$

Then, 


$$
\begin{aligned}
& V_{T 3}(\cdot)=\left(S(t) \cdot e^{\Sigma}-X_{e}\right)\left(1-\frac{\beta_{0} \cdot X_{e}}{X_{e}+\delta}\right)-\frac{\left(\beta-\beta_{0}\right) \cdot X_{e}}{\ln (1+\alpha)}\left[\frac{S(t) \cdot e^{\Sigma}}{X_{e}+\delta}-\ln \left(\frac{S(t) \cdot e^{\Sigma}}{X_{e}+\delta}\right)\right]+\frac{\left(\beta-\beta_{0}\right) \cdot X_{e}}{\ln (1+\alpha)}+\frac{\beta_{0} \cdot X_{e} \cdot \delta}{X_{e}+\delta} \\
& =\left[\left(1-\frac{\beta_{0} \cdot X_{e}}{X_{e}+\delta}\right)-\frac{\left(\beta-\beta_{0}\right) \cdot X_{e}}{\ln (1+\alpha) \cdot\left(X_{e}+\delta\right)}\right] \cdot S(t) \cdot e^{\Sigma}+\frac{\left(\beta-\beta_{0}\right) \cdot X_{e}}{\ln (1+\alpha)} \sigma \sqrt{T-t} \cdot Z \\
& +\frac{\left(\beta-\beta_{0}\right) \cdot X_{e}}{\ln (1+\alpha)}\left[\ln S(t)+(T-t)\left(r-\frac{\sigma^{2}}{2}\right)\right] \\
& -X_{e}\left(1-\frac{\beta_{0} \cdot X_{e}}{X_{e}+\delta}\right)-\frac{\left(\beta-\beta_{0}\right) \cdot X_{e}}{\ln (1+\alpha)} \ln \left(X_{e}+\delta\right)+\frac{\left(\beta-\beta_{0}\right) \cdot X_{e}}{\ln (1+\alpha)}+\frac{\beta_{0} \cdot X_{e} \cdot \delta}{X_{e}+\delta} \\
& =\left[\left(1-\frac{\beta_{0} \cdot X_{e}}{X_{e}+\delta}\right)-\frac{\left(\beta-\beta_{0}\right) \cdot X_{e}}{\ln (1+\alpha) \cdot\left(X_{e}+\delta\right)}\right] \cdot S(t) \cdot e^{\Sigma}+\frac{\left(\beta-\beta_{0}\right) \cdot X_{e}}{\ln (1+\alpha)} \sigma \sqrt{T-t} \cdot Z \\
& +\frac{\beta_{0} \cdot X_{e} \cdot\left(\delta+X_{e}\right)}{X_{e}+\delta}-X_{e}+\frac{\left(\beta-\beta_{0}\right) \cdot X_{e}}{\ln (1+\alpha)}\left[1+\ln S(t)+(T-t)\left(r-\frac{\sigma^{2}}{2}\right)-\ln \left(X_{e}+\delta\right)\right] \\
& =\left[\left(1-\frac{\beta_{0} \cdot X_{e}}{X_{e}+\delta}\right)-\frac{\left(\beta-\beta_{0}\right) \cdot X_{e}}{\ln (1+\alpha) \cdot\left(X_{e}+\delta\right)}\right] \cdot S(t) \cdot e^{\Sigma}+\frac{\left(\beta-\beta_{0}\right) \cdot X_{e}}{\ln (1+\alpha)} \sigma \sqrt{T-t} \cdot Z \\
& +\left(\beta_{0}-1\right) \cdot X_{e}+\frac{\left(\beta-\beta_{0}\right) \cdot X_{e}}{\ln (1+\alpha)}\left[1+\ln \left(\frac{S(t)}{X_{e}+\delta}\right)+(T-t)\left(r-\frac{\sigma^{2}}{2}\right)\right] .
\end{aligned}
$$

If

$$
\begin{aligned}
& M=S(t) \cdot\left[1-\frac{\beta_{0} \cdot X_{e}}{X_{e}+\delta}-\frac{\left(\beta-\beta_{0}\right) \cdot X_{e}}{\left(X_{e}+\delta\right) \cdot \ln (1+\alpha)}\right] \\
& H=\frac{\left(\beta-\beta_{0}\right) \cdot X_{e}}{\ln (1+\alpha)} \cdot \sigma \sqrt{T-t} \\
& I=\left(\beta_{0}-1\right) \cdot X_{e}+\frac{\left(\beta-\beta_{0}\right) \cdot X_{e}}{\ln (1+\alpha)}\left[1+(T-t)\left(r-\frac{\sigma^{2}}{2}\right)+\ln \left(\frac{S(t)}{X_{e}+\delta}\right)\right],
\end{aligned}
$$

then $V_{T 3}(\cdot)$ can be further simplified into

$$
V_{T 3}(\cdot)=M \cdot e^{\Sigma}+H \cdot Z+I \text {. }
$$

$$
\begin{aligned}
V_{3}(S(t), t) & =\frac{e^{-r(T-t)}}{\sqrt{2 \pi}} \int_{X_{e}+\delta \leq S(t)<(1+\alpha)\left(X_{e}+\delta\right)}\left(M \cdot e^{\Sigma}+H \cdot Z+I\right) \cdot e^{-\left(Z^{2} / 2\right)} \mathrm{d} Z \\
& =\frac{e^{-r(T-t)}}{\sqrt{2 \pi}} \int_{d_{2}}^{d_{3}} M \cdot e^{\Sigma} \cdot e^{-\left(Z^{2} / 2\right)} \mathrm{d} Z+\frac{e^{-r(T-t)}}{\sqrt{2 \pi}} \int_{d_{2}}^{d_{3}} H \cdot Z \cdot e^{-\left(Z^{2} / 2\right)} \mathrm{d} Z+\frac{e^{-r(T-t)}}{\sqrt{2 \pi}} \int_{d_{2}}^{d_{3}} I \cdot e^{-\left(Z^{2} / 2\right)} \mathrm{d} Z \\
& =\frac{M}{\sqrt{2 \pi}} \int_{d_{2}}^{d_{3}} e^{-1 / 2(Z-\sqrt{T-t})^{2}} \mathrm{~d} Z-\frac{e^{-r(T-t)} \cdot H}{\sqrt{2 \pi}}\left(e^{-\left(d_{3}^{2} / 2\right)}-e^{-\left(d_{2}^{2} / 2\right)}\right)+e^{-r(T-t)} \cdot I \cdot\left(N\left(d_{3}\right)-N\left(d_{2}\right)\right)
\end{aligned}
$$




$$
\begin{aligned}
& =M \cdot\left(N\left(d_{6}\right)-N\left(d_{5}\right)\right)-\frac{e^{-r(T-t)} \cdot H}{\sqrt{2 \pi}}\left(e^{-\left(d_{3}^{2} / 2\right)}-e^{-\left(d_{2}^{2} / 2\right)}\right)+e^{-r(T-t)} \cdot I \cdot\left(N\left(d_{3}\right)-N\left(d_{2}\right)\right) \\
V_{T 4}(\cdot) & =\left(S(t) \cdot e^{\Sigma}-X_{e}\right)\left(1-\frac{\beta_{0} \cdot X_{e}}{X_{e}+\delta}\right)-\frac{\alpha \cdot\left(\beta-\beta_{0}\right) \cdot X_{e} \cdot S(t) \cdot e^{\Sigma}}{\left(X_{e}+\delta\right) \cdot(1+\alpha) \cdot \ln (1+\alpha)}+X_{e} \cdot\left(\beta-\beta_{0}\right)+\frac{\beta_{0} \cdot X_{e} \cdot \delta}{X_{e}+\delta} \\
& =\left(1-\frac{\beta_{0} \cdot X_{e}}{X_{e}+\delta}-\frac{\alpha \cdot\left(\beta-\beta_{0}\right) \cdot X_{e}}{\left(X_{e}+\delta\right) \cdot(1+\alpha) \cdot \ln (1+\alpha)}\right) \cdot S(t) \cdot e^{\Sigma}-(1-\beta) \cdot X_{e} .
\end{aligned}
$$

If

$$
\begin{aligned}
& Q=1-\frac{\beta_{0} \cdot X_{e}}{X_{e}+\delta}-\frac{\alpha \cdot\left(\beta-\beta_{0}\right) \cdot X_{e}}{\left(X_{e}+\delta\right) \cdot(1+\alpha) \cdot \ln (1+\alpha)} \\
& G=(1-\beta) \cdot X_{e},
\end{aligned}
$$

then $\mathrm{V}_{T 4}(\cdot)$ can be further simplified into

$$
\mathrm{V}_{T 4}(\cdot)=Q \cdot S(t) \cdot e^{\Sigma}-G \text {. }
$$

$$
\begin{aligned}
V_{4}(S(t), t) & =\frac{e^{-r(T-t)}}{\sqrt{2 \pi}} \int_{S(t) \geq(1+\alpha)\left(X_{e}+\delta\right)}^{+\infty}\left(Q \cdot S(t) \cdot e^{\Sigma}-G\right) \cdot e^{-\left(Z^{2} / 2\right)} \cdot \mathrm{d} Z \\
& =\frac{e^{-r(T-t)}}{\sqrt{2 \pi}} \int_{d_{3}}^{+\infty}\left(Q \cdot S(t) \cdot e^{\Sigma}\right) \cdot e^{-\left(Z^{2} / 2\right)} \cdot \mathrm{d} Z-\frac{e^{-r(T-t)}}{\sqrt{2 \pi}} \int_{d_{3}}^{+\infty} G \cdot e^{-\left(Z^{2} / 2\right)} \cdot \mathrm{d} Z \\
& =\frac{Q \cdot S(t)}{\sqrt{2 \pi}} \int_{d_{3}-\sigma \sqrt{T-t}}^{+\infty} e^{-\left((Z-\sigma \sqrt{T-t})^{2} / 2\right)} \cdot d(Z-\sigma \sqrt{T-t})-\frac{G \cdot e^{-r(T-t)}}{\sqrt{2 \pi}} \int_{d_{3}}^{+\infty} e^{-\left(Z^{2} / 2\right)} \cdot \mathrm{d} Z \\
& =Q \cdot S(t) \cdot\left(1-N\left(d_{6}\right)\right)-G \cdot e^{-r(T-t)}\left(1-N\left(d_{3}\right)\right) .
\end{aligned}
$$

Consequently, the final pricing formula for the proactive hedging call option is

$$
\begin{aligned}
V\left(S(t), t, \alpha, \beta_{0}, \beta, \delta\right)= & V_{C}(S(t), t) \\
= & V_{1}(S(t), t)+V_{2}(S(t), t)+V_{3}(S(t), t)+V_{4}(S(t), t) \\
= & S(t) \cdot\left(N\left(d_{5}\right)-N\left(d_{4}\right)\right)-X_{e} \cdot e^{-r(T-t)}\left(N\left(d_{2}\right)-N\left(d_{1}\right)\right)+M \cdot\left(N\left(d_{6}\right)-N\left(d_{5}\right)\right) \\
& -\frac{e^{-r(T-t)} \cdot H}{\sqrt{2 \pi}}\left(e^{-\left(d_{3}^{2} / 2\right)}-e^{-\left(d_{2}^{2} / 2\right)}\right)+e^{-r(T-t)} \cdot I \cdot\left(N\left(d_{3}\right)-N\left(d_{2}\right)\right)+Q \cdot S(t) \cdot\left(1-N\left(d_{6}\right)\right) \\
& -G \cdot e^{-r(T-t)}\left(1-N\left(d_{3}\right)\right),
\end{aligned}
$$

where 


$$
\begin{aligned}
& d_{1}=\frac{\ln X_{e} / S-(T-t)\left(r-\sigma^{2} / 2\right)}{\sigma \sqrt{T-t}} \\
& d_{2}=\frac{\ln X_{e}+\delta / S-(T-t)\left(r-\sigma^{2} / 2\right)}{\sigma \sqrt{T-t}} \\
& d_{3}=\frac{\ln (1+\alpha)\left(X_{e}+\delta\right) / S-(T-t)\left(r-\sigma^{2} / 2\right)}{\sigma \sqrt{T-t}}, \\
& d_{4}=d_{1}-\sigma \sqrt{T-t}=\frac{\ln X_{e} / S-(T-t)\left(r+\sigma^{2} / 2\right)}{\sigma \sqrt{T-t}}, \\
& d_{5}=d_{2}-\sigma \sqrt{T-t}=\frac{\ln X_{e}+\delta / S-(T-t)\left(r+\sigma^{2} / 2\right)}{\sigma \sqrt{T-t}}, \\
& d_{6}=d_{3}-\sigma \sqrt{T-t}=\frac{\ln (1+\alpha)\left(X_{e}+\delta\right) / S-(T-t)\left(r+\sigma^{2} / 2\right)}{\sigma \sqrt{T-t}}, \\
& M=S(t) \cdot\left[1-\frac{\beta_{0} \cdot X_{e}}{X_{e}+\delta}-\frac{\left(\beta-\beta_{0}\right) \cdot X_{e}}{\left(X_{e}+\delta\right) \cdot \ln (1+\alpha)}\right], \\
& H=\frac{\left(\beta-\beta_{0}\right) \cdot X_{e}}{\ln (1+\alpha)} \cdot \sigma \sqrt{T-t}, \\
& I=\left(\beta_{0}-1\right) \cdot X_{e}+\frac{\left(\beta-\beta_{0}\right) \cdot X_{e}}{\ln (1+\alpha)}\left[1+(T-t)\left(r-\frac{\sigma^{2}}{2}\right)+\ln \left(\frac{S(t)}{X_{e}+\delta}\right)\right], \\
& Q=1-\frac{\beta_{0} \cdot X_{e}}{X_{e}+\delta}-\frac{\alpha \cdot\left(\beta-\beta_{0}\right) \cdot X_{e}}{\left(X_{e}+\delta\right) \cdot(1+\alpha) \cdot \ln (1+\alpha)}, \\
& G=(1-\beta) \cdot X_{e} .
\end{aligned}
$$

At this point, the pricing formula for a proactive hedging call option with a linear position strategy is obtained.

\section{Pricing Formula Discussion for Selected Special Cases}

This section presents and discusses several special cases involving exotic options. The pricing formula in equation (41) has two sets of parameters. The first set includes $\beta, \beta_{0}, \delta$, and $\alpha$, which describe the general logarithmic position strategy. The second set includes $r, T, \sigma$, and $X_{e}$, which are the basic parameters for the pricing of a classical
European option. Herein, we focus on two special cases based on the first set of parameters:

Special Case 1: when $\beta=\beta_{0}=0, \delta=0$, and $\alpha>0$, this means that the option holder does not trade the underlying asset during the entire holding period. Theoretically, this exotic option pricing should be consistent with the classical European option pricing. Under this condition, we can define the following: $M=S(t), H=0, I=-X_{e}, Q=1, G=X_{e}$

Then,

$$
\begin{aligned}
V(S(t), t, \alpha, 0,0,0)= & V_{C}(S(t), t) \\
= & V_{1}(S(t), t)+V_{2}(S(t), t)+V_{3}(S(t), t)+V_{4}(S(t), t) \\
= & S(t)\left(N\left(d_{5}\right)-N\left(d_{4}\right)\right)-X_{e} \cdot e^{-r(T-t)}\left(N\left(d_{2}\right)-N\left(d_{1}\right)\right)+S(t) \cdot\left(N\left(d_{6}\right)-N\left(d_{5}\right)\right) \\
& -X_{e} \cdot e^{-r(T-t)} \cdot\left(N\left(d_{3}\right)-N\left(d_{2}\right)\right)+S(t) \cdot\left(1-N\left(d_{6}\right)\right)-X_{e} \cdot e^{-r(T-t)}\left(1-N\left(d_{3}\right)\right) \\
= & S(t)\left(N\left(d_{5}\right)-N\left(d_{4}\right)+N\left(d_{6}\right)-N\left(d_{5}\right)+1-N\left(d_{6}\right)\right) \\
& -X_{e} \cdot e^{-r(T-t)}\left(N\left(d_{2}\right)-N\left(d_{1}\right)+N\left(d_{3}\right)-N\left(d_{2}\right)+1-N\left(d_{3}\right)\right) \\
= & S(t)\left(N\left(-d_{4}\right)\right)-X_{e} \cdot e^{-r(T-t)}\left(N\left(-d_{1}\right)\right) .
\end{aligned}
$$


It is clear that equation (43) is consistent with the results obtained using the Black-Scholes formula.

Special Case 2: when $\beta=\beta_{0}=1, \delta=0, \alpha=\varepsilon$, and $\varepsilon>0$ (where $\varepsilon$ is a very small number), this means that traders use all of their money to buy underlying assets when the underlying asset price is above but very close to $X_{e}$. Then, theoretically, this position strategy almost completely covers the risk exposure of the underlying asset, which means that the value of this exotic option is close to 0 .

Under this condition, we can define the following: $M=0$, $H=0, I=0, Q=0, G=0, d_{0}=d_{1}=d_{2}, d=d_{3}=d_{4}$

Then,

$$
\begin{aligned}
V(S(t), t, \alpha, 1,1,0)= & V_{C}(S(t), t) \\
= & S(t) \cdot\left(N\left(d_{5}\right)-N\left(d_{4}\right)\right) \\
& -X_{e} \cdot e^{-r(T-t)}\left(N\left(d_{2}\right)-N\left(d_{1}\right)\right) \\
= & S(t)\left(N\left(d_{5}\right)-N\left(d_{4}\right)+N\left(d_{6}\right)\right. \\
& \left.-N\left(d_{5}\right)+1-N\left(d_{6}\right)\right) \\
= & 0 .
\end{aligned}
$$

\section{Comparing Theoretical Prices of Proactive Hedging Call Options with General Logarithmic vs. General Linear Position Strategies}

To verify that the exotic option proposed in this study has a price advantage, a simulation method was applied to compare the difference in the theoretical price of the European option with a general logarithmic position strategy, the European option with a linear strategy, and the classical European option. The pricing formula for the European option with the linear position strategy was described in a report by Wang et al. [15]. To facilitate this comparison, the parameter symbols used in this study are consistent with those used by Wang et al. [15] and in the classical Black-Scholes pricing formula. The calculation parameters used in this study are shown in Table 1. For simplicity, we assume $t=0$ across all calculations in this section.

The dynamic position strategy parameters and the basic parameters for the classic European option in Table 1 were used to generate several potential parameter combinations. Then, the corresponding pricing formulas were used to calculate the theoretical prices of the European option with a general logarithmic position strategy, the European option with a linear position strategy, and the classical European option. The theoretical prices of these calculated options are listed in Table 2. To intuitively observe the price advantages of the exotic options proposed in this study, Table 2 includes the price ratios, which are defined by the following relationship:

$$
\text { ratio }=\frac{P_{\text {exotic option }}}{P_{\text {classical option }}} \text {. }
$$

TABLE 1: Calculation parameters.

\begin{tabular}{lc}
\hline Symbol & Values \\
\hline$T$ & 0 \\
$\alpha$ & $50 \%, 70 \%, 100 \%$ \\
$\beta$ & $60 \%, 100 \%$ \\
$\beta_{0}$ & $30 \%, 50 \%$ \\
$\delta$ & $\$ 0, \$ 7, \$ 15$ per share \\
$T$ & 0.5 (half of one year) \\
$S_{0}$ & $\$ 20$ per share \\
$X_{e}$ & $\$ 20$ per share \\
$\sigma$ & $30 \%, 20 \%, 10 \%$ \\
$r$ & $6 \%$ \\
\hline
\end{tabular}

The results in Table 2 have several important indications:

(1) The theoretical prices of the proactive hedging European option are cheaper than those of the analogous classical European option under all tested parameter combinations. Moreover, the greater the market volatility is (i.e., the greater the value of $\sigma$ ), the greater the price advantage of the exotic option is. As shown in Table 2, when the market volatility is $\sigma=30 \%$, the price of the proactive hedging European option with a general logarithmic position strategy (i.e., $\alpha=0.5, \beta=1, \beta_{0}=0.5, \delta=0$ ) is only $34 \%$ of the price of the classical European option.

(2) The theoretical price of the proactive hedging European option with a general logarithmic position strategy is lower than that with a general linear position strategy. This result indicates that the general logarithmic position strategy would likely be more attractive to hedgers. This price advantage is especially pronounced when $\delta=0$. In this case, when the market volatility is $\sigma=30 \%$ and the other strategy parameters are $\alpha=0.5, \beta=1$, and $\beta_{0}=0.5$, the European option with a general logarithmic position strategy can save $5.5 \%$ more of the option premium than the linear position strategy. This means that the price advantage of the European option with the general logarithmic position strategy mainly depends on $\delta$.

(3) Each of the four strategy parameters $\left(\alpha, \beta, \beta_{0}\right.$, and $\left.\delta\right)$ has a remarkable impact on the price advantage. The results in Table 2 show that when other variables are the same, the smaller the values of $\alpha$ and $\delta$ (where $0.5 \leq \alpha \leq 1)$, or the larger the values of $\beta$ and $\beta_{0}$ $\left(0 \leq \beta_{0} \leq \beta \leq 1\right)$, and the greater the price advantage of the European option with the general logarithmic position strategy. This implies that the price advantage of these exotic options substantially depends on the amount of capital that is used to execute the dynamic position strategy.

To evaluate the effects of changes in the strategy parameters $\left(\alpha, \delta, \beta_{0}\right.$, and $\left.\beta\right)$ on the theoretical price of options, the observed parameters were set as continuous variables, and all other strategy parameters were set as fixed values. According to the conclusions described above, the smaller 
TABLE 2: Price ratios of exotic options considering the proactive hedging options relative to classical European options in terms of $\alpha, \beta, \beta_{0}, \delta$, and $\sigma$ parameters.

\begin{tabular}{|c|c|c|c|c|c|c|c|c|c|}
\hline \multirow{2}{*}{\multicolumn{4}{|c|}{ Strategy parameters }} & \multicolumn{6}{|c|}{ Price ratios of exotic options with classical option } \\
\hline & & & & \multicolumn{2}{|c|}{$\sigma=30 \%$} & \multicolumn{2}{|c|}{$\sigma=20 \%$} & \multicolumn{2}{|c|}{$\sigma=10 \%$} \\
\hline$\alpha$ & $\beta_{0}$ & $\beta$ & $\delta$ & Logarithmic & Linear & Logarithmic & Linear & Logarithmic & Linear \\
\hline 0.5 & 0.3 & 0.6 & 0 & 0.60 & 0.61 & 0.63 & 0.64 & 0.66 & 0.67 \\
\hline 0.5 & 0.3 & 0.6 & 7 & 0.97 & 0.97 & 0.99 & 0.99 & 1.00 & 1.00 \\
\hline 0.5 & 0.3 & 0.6 & 15 & 1.00 & 1.00 & 1.00 & 1.00 & 1.00 & 1.00 \\
\hline 0.5 & 0.5 & 0.6 & 0 & 0.47 & 0.47 & 0.48 & 0.48 & 0.49 & 0.49 \\
\hline 0.5 & 0.5 & 0.6 & 7 & 0.96 & 0.96 & 0.99 & 0.99 & 1.00 & 1.00 \\
\hline 0.5 & 0.5 & 0.6 & 15 & 1.00 & 1.00 & 1.00 & 1.00 & 1.00 & 1.00 \\
\hline 0.5 & 0.5 & 1 & 0 & 0.34 & 0.36 & 0.39 & 0.40 & 0.44 & 0.45 \\
\hline 0.5 & 0.5 & 1 & 7 & 0.95 & 0.95 & 0.99 & 0.99 & 1.00 & 1.00 \\
\hline 0.5 & 0.5 & 1 & 15 & 1.00 & 1.00 & 1.00 & 1.00 & 1.00 & 1.00 \\
\hline 0.7 & 0.3 & 0.6 & 0 & 0.63 & 0.64 & 0.65 & 0.66 & 0.67 & 0.68 \\
\hline 0.7 & 0.3 & 0.6 & 7 & 0.97 & 0.97 & 0.99 & 0.99 & 1.00 & 1.00 \\
\hline 0.7 & 0.3 & 0.6 & 15 & 1.00 & 1.00 & 1.00 & 1.00 & 1.00 & 1.00 \\
\hline 0.7 & 0.5 & 0.6 & 0 & 0.48 & 0.48 & 0.48 & 0.49 & 0.49 & 0.49 \\
\hline 0.7 & 0.5 & 0.6 & 7 & 0.96 & 0.96 & 0.99 & 0.99 & 1.00 & 1.00 \\
\hline 0.7 & 0.5 & 0.6 & 15 & 1.00 & 1.00 & 1.00 & 1.00 & 1.00 & 1.00 \\
\hline 0.7 & 0.5 & 1 & 0 & 0.38 & 0.40 & 0.41 & 0.43 & 0.45 & 0.46 \\
\hline 0.7 & 0.5 & 1 & 7 & 0.95 & 0.95 & 0.99 & 0.99 & 1.00 & 1.00 \\
\hline 0.7 & 0.5 & 1 & 15 & 1.00 & 1.00 & 1.00 & 1.00 & 1.00 & 1.00 \\
\hline 1 & 0.3 & 0.6 & 0 & 0.64 & 0.66 & 0.66 & 0.67 & 0.68 & 0.68 \\
\hline 1 & 0.3 & 0.6 & 7 & 0.97 & 0.97 & 0.99 & 0.99 & 1.00 & 1.00 \\
\hline 1 & 0.3 & 0.6 & 15 & 1.00 & 1.00 & 1.00 & 1.00 & 1.00 & 1.00 \\
\hline 1 & 0.5 & 0.6 & 0 & 0.48 & 0.49 & 0.49 & 0.49 & 0.49 & 0.50 \\
\hline 1 & 0.5 & 0.6 & 7 & 0.96 & 0.96 & 0.99 & 0.99 & 1.00 & 1.00 \\
\hline 1 & 0.5 & 0.6 & 15 & 1.00 & 1.00 & 1.00 & 1.00 & 1.00 & 1.00 \\
\hline 1 & 0.5 & 1 & 0 & 0.41 & 0.43 & 0.43 & 0.45 & 0.46 & 0.47 \\
\hline 1 & 0.5 & 1 & 7 & 0.95 & 0.95 & 0.99 & 0.99 & 1.00 & 1.00 \\
\hline 1 & 0.5 & 1 & 15 & 1.00 & 1.00 & 1.00 & 1.00 & 1.00 & 1.00 \\
\hline
\end{tabular}

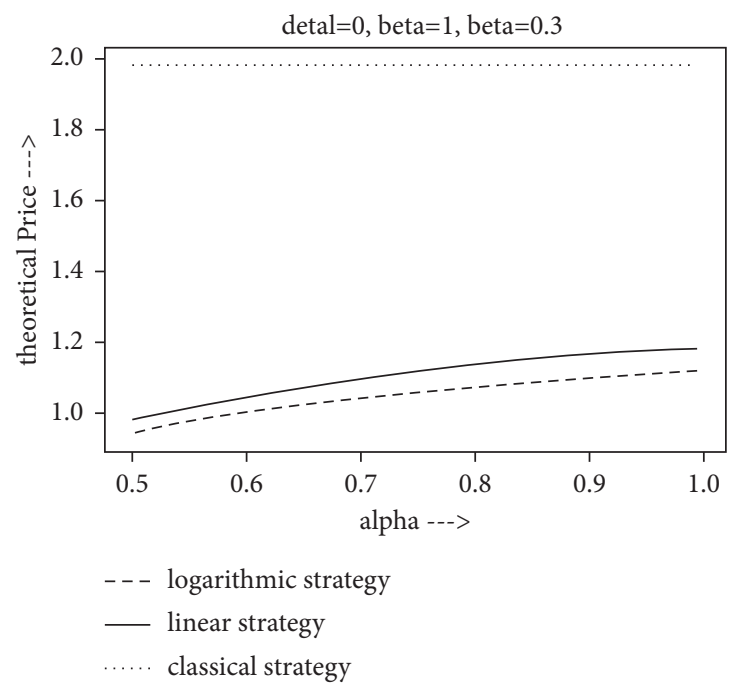

(a)

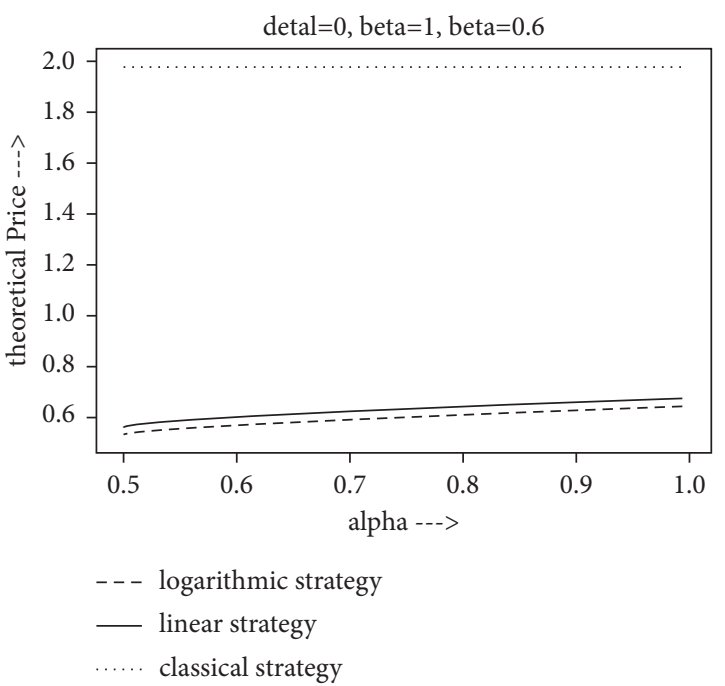

(b)

Figure 3: Continued. 


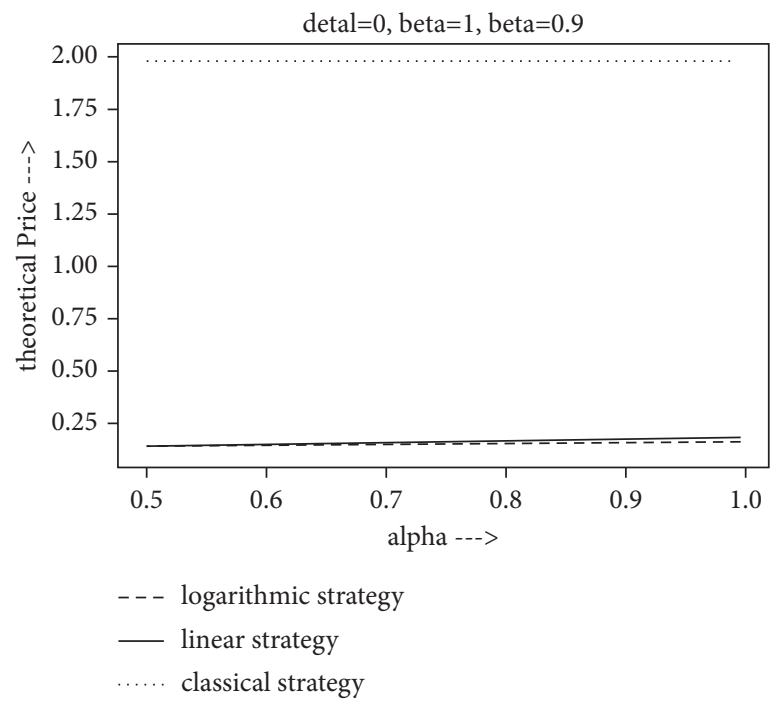

(c)

Figure 3: The theoretical price as a function of $\alpha$ when $\delta=0, \beta=1$, and $\beta_{0}=0.3,0.6$, or 0.9 (a, b, c), respectively.

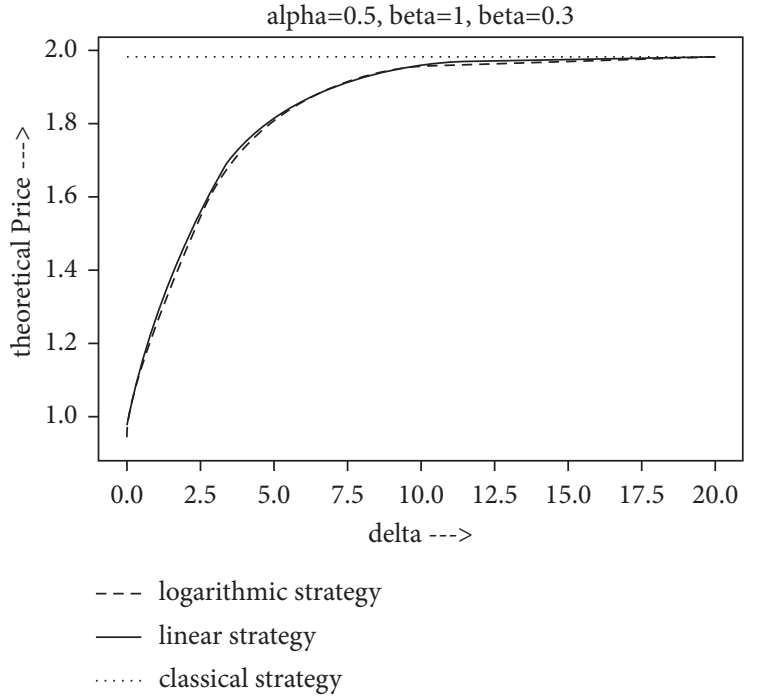

(a)

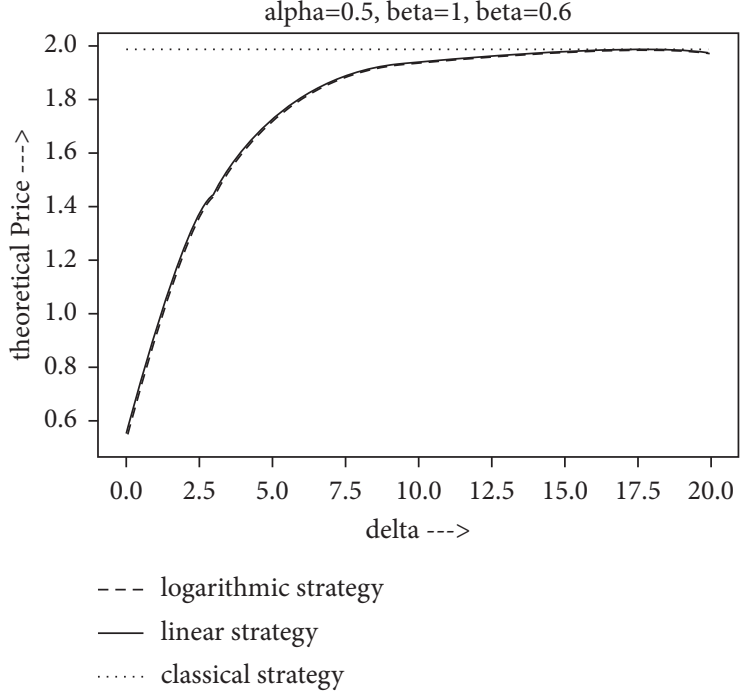

(b)

Figure 4: Continued. 


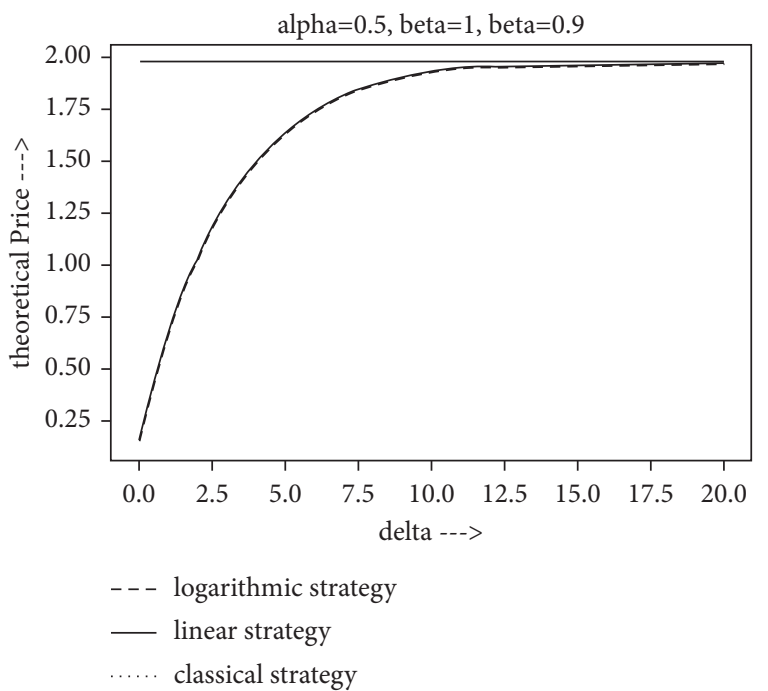

(c)

Figure 4: The theoretical price as a function of $\delta$ when $\alpha=0.5, \beta=1$, and $\beta_{0}=0.3,0.6$, or 0.9 (a, b, c), respectively.

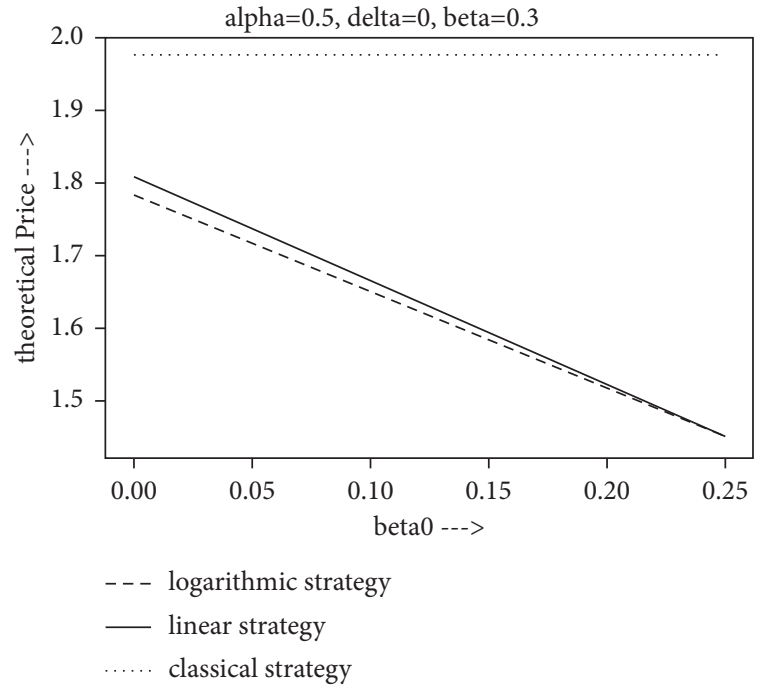

(a)

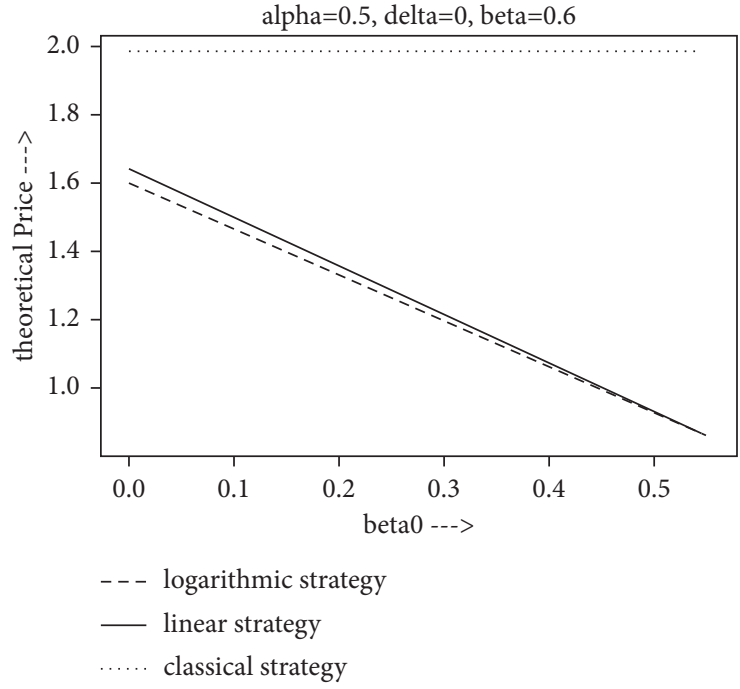

(b)

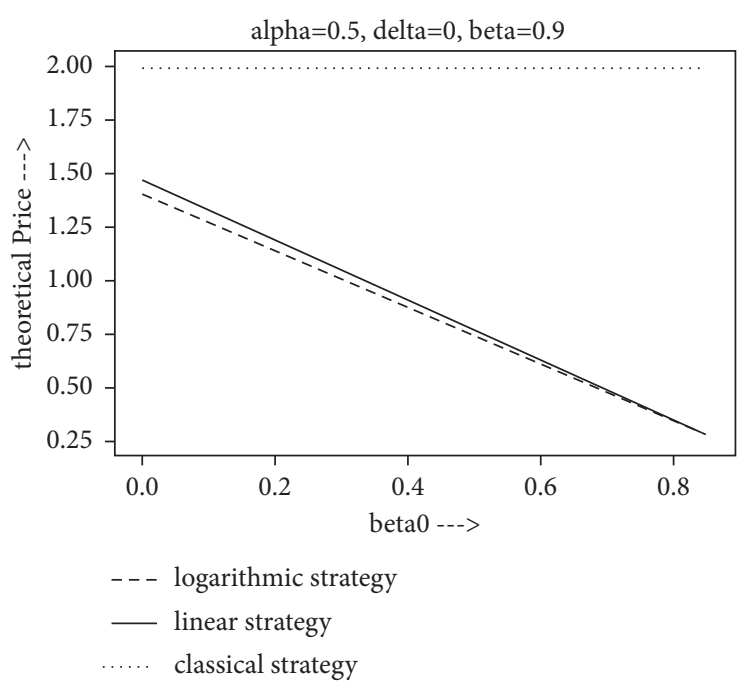

(c)

Figure 5: The theoretical price as a function of $\beta_{0}$ when $\alpha=0.5, \delta=0$, and $\beta=0.3,0.6$, or 0.9 (a, b, c), respectively. 

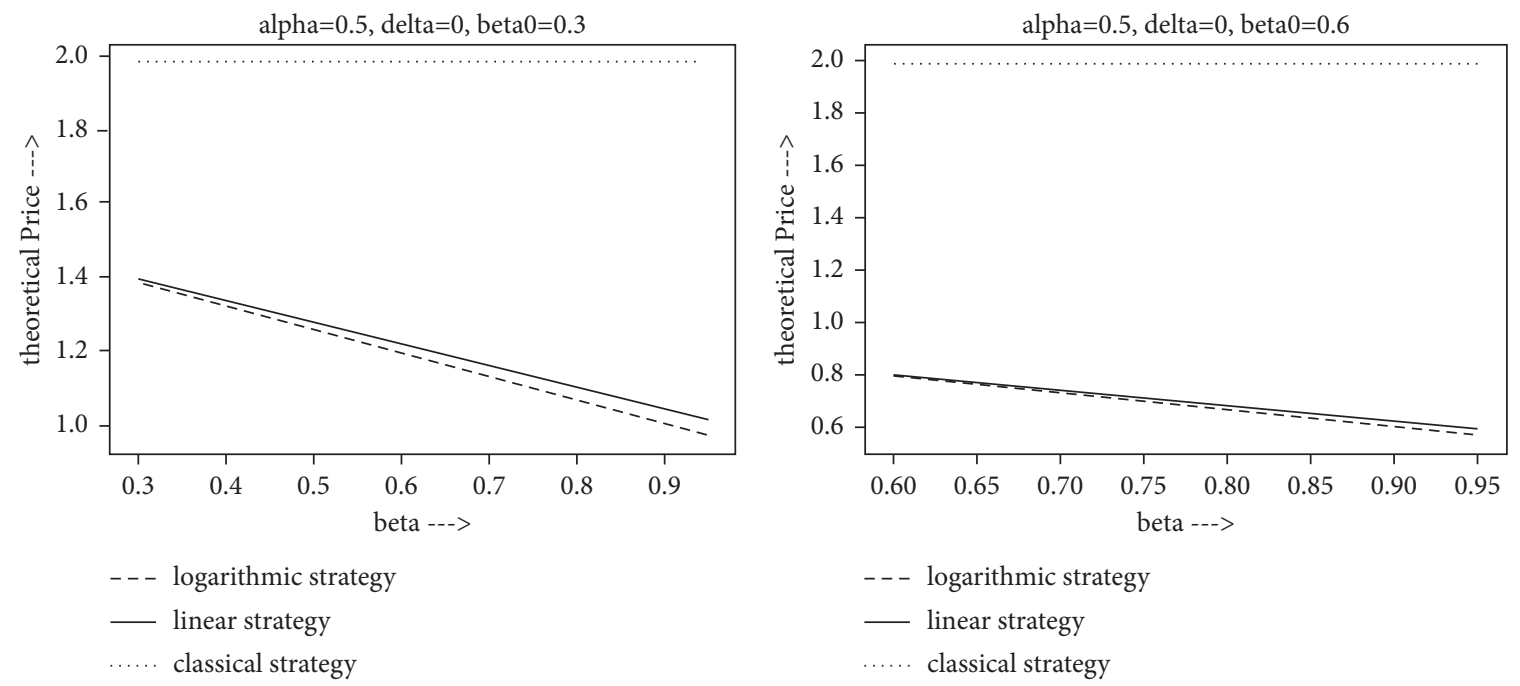

(a)

(b)

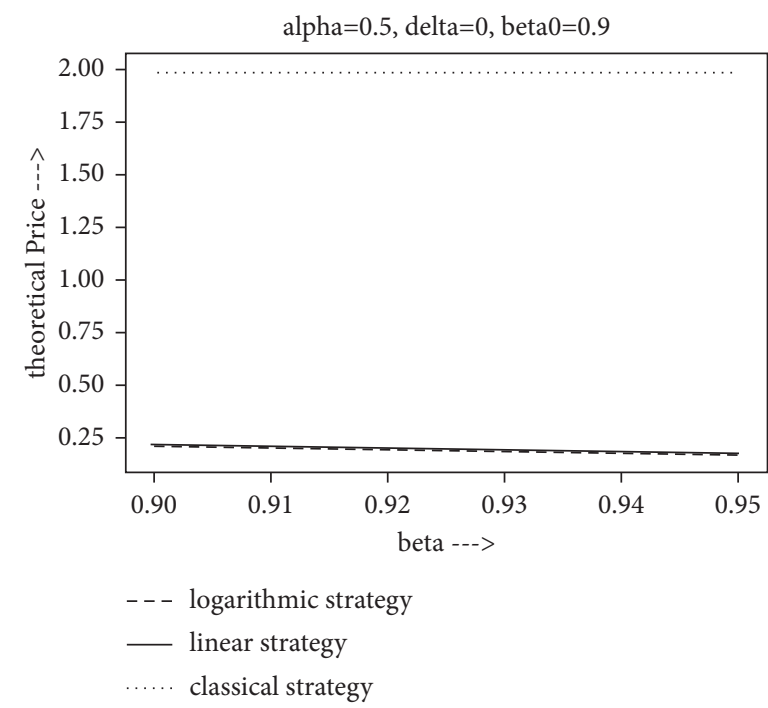

(c)

Figure 6: The theoretical price as a function of $\beta$ when $\alpha=0.5, \delta=0$, and $\beta_{0}=0.3,0.6$, or 0.9 (a, b, c), respectively.

the $\alpha$ and $\delta$ values, or the larger the $\beta_{0}$ and $\beta$ values, the greater the price advantage of this exotic option; therefore, we set the fixed parameter values to correspond to the situation with the greatest price advantage $(\alpha=0.5, \delta=0$, $\beta=1$ ) and compared this exotic option with the classical European call option.

If the interval of $\alpha \in[0.5,1]$ holds true (Figure 3 ), the price advantage of this general logarithmic position strategy is the largest when $\alpha=0.5$. As $\alpha$ increases, the price advantage of this exotic option decreases. However, the slope of the curve is not steep, which indicates that $\alpha$ has a relatively small influence on the price of the option.

If the interval of $\delta \in\left[0, X_{e}=20\right]$ holds true (Figure 4), the price advantage of the option is the largest when $\delta=0$. As $\delta$ increases, the price advantage of this exotic option decreases. In contrast to the $\alpha$ effect, the $\delta$ curve is clearly convex, which means that the closer $\delta$ is to 0 , the more sensitive this exotic option price is to the $\delta$ parameter. This $\delta$ effect becomes extremely weak as $\delta$ approaches $1 / 2 X_{e}=10$.

The impacts of the $\beta_{0}$ and $\beta$ parameters are presented in Figures 3-6, and Figures 5 and 6 , in particular, illustrate that the larger the values of $\beta_{0}$ and $\beta$, the greater the price advantage of the exotic option. If $0 \leq \beta_{0} \leq \beta \leq 1$, Figures 5 and 6 both indicate that $\beta_{0}$ and $\beta$ follow monotonic, linearly decreasing functions. If all other parameters are equal, the linear slope of $\beta_{0}$ is steeper than that of $\beta$, which means that the decreasing effect of $\beta_{0}$ is more significant. In addition, the trends in Figures 3-6 demonstrate that the smaller the value of $\beta_{0}$, the more advantageous the general logarithmic position strategy. 


\section{Conclusions}

This study details and discusses the pricing of an exotic option, which requires the call option buyer to actively cover the risk exposure by trading the underlying asset according to a preagreed general logarithmic position strategy. The option seller only bears the losses resulting from uncovered risk exposure. To test the pricing advantage of this exotic option, its pricing was compared with that of the linear position strategy proposed by Wang et al. [13-15] and that of the classical European option.

The comparative analysis presented herein revealed that both of the described proactive hedging European options exhibited significant price advantages over the classical European option. For example, the theoretical price of applying the general logarithmic position strategy was as low as 34\% of the classical European option price under certain parameter settings. The price advantage of this exotic option mainly depended on the capital utilization coefficients $(\beta$, $\beta_{0}$ ), which were used to actively hedge the exposure risk of the underlying asset and the prices of the underlying asset $\left(X_{e}+\delta\right)$ when the proactive hedging strategy was initiated. Both $\beta_{0}$ and $\beta$ are monotonic, linearly decreasing functions of the exotic option, meaning that the larger the values of $\beta_{0}$ and $\beta$, the greater the price advantage of this exotic option. Especially when $\beta_{0}$ is very small, the European option with a general logarithmic position strategy has a considerable price advantage over that of the linear position strategy, and its implementation can save up to $5.5 \%$ of the option premiums. Additionally, this exotic option price is more sensitive to changes in the $\delta$ parameter as $\delta$ approaches zero; the $\delta$ effect becomes weaker as $\delta$ approaches $1 / 2 X_{e}=10$. These results indicate that it is more suitable for traders to use the European option with a general logarithmic position strategy when the initial amount of capital is limited.

This study derives the theoretical price of the proactive hedging European option by applying a general logarithmic dynamic position strategy as a representative nonlinear position strategy. The described analyses indicate that the extension of this exotic option with the general logarithmic position strategy has important practical significance. Further studies should aim to develop more reasonable dynamic position strategies in the form of nonlinear functions.

\section{Data Availability}

The simulation codes and data used to support the findings of this study are available from the corresponding author upon request.

\section{Conflicts of Interest}

The authors declare that they have no conflicts of interest.

\section{Acknowledgments}

This research was funded by the National Science Foundation of China (nos. 71503060 and 71532004 and 72071038) and the Ministry of Education in China Project of Humanities and Social Sciences (no. 14YJC790065).

\section{References}

[1] H. Yor and M. Yor, "Bessel processes, Asian options, and perpetuities," Mathematical Finance, vol. 3, no. 4, pp. 349-375, 1993.

[2] A. Horvath and P. Medvegyev, "Pricing asian options: a comparison of numerical and simulation approaches twenty years later," Journal of Mathematical Finance, vol. 6, pp. 10-841, 2016.

[3] M. B. Goldman, H. B. Sosin, and M. A. Gatto, "Path dependent options: "buy at the low, sell at the high" path dependent options: "buy at the low, sell at the high"," The Journal of Finance, vol. 34, no. 5, pp. 1111-1127, 1979.

[4] G. Ahmad and O. Nikan, "A computational method based on the moving least-squares approach for pricing double barrier options in a time-fractional black-scholes model," Computational Economics, vol. 55, pp. 119-141, 2020.

[5] J. H. Guo and L. F. Chang, "Repeated Richardson extrapolation and static hedging of barrier options under the CEV model," Journal of Futures Markets, vol. 40, no. 6, pp. 974-988, 2020.

[6] V. Linetsky, "Step options," Mathematical Finance, vol. 9, no. 1, pp. 55-96, 1999.

[7] J. Detemple, S. Laminou Abdou, and F. Moraux, "American step options," European Journal of Operational Research, vol. 282, no. 1, pp. 363-385, 2020.

[8] E. Singirankabo, P. Ngare, and C. Ogutu, "Pricing lookback option using multinomial lattice," Mathematical Finance, vol. 9, no. 1, pp. 1-12, 2020.

[9] H. He, W. P. Keirstead, and J. Rebholz, "Double lookbacks," Mathematical Finance, vol. 8, no. 3, pp. 201-228, 1998.

[10] N. Tahani, "Exotic geometric average options pricing under stochastic volatility," Applied Mathematical Finance, vol. 20, no. 3, pp. 229-245, 2013.

[11] Y. Muroi, "Pricing lookback options with knock-out boundaries," Applied Mathematical Finance, vol. 13, no. 2, pp. 155-190, 2006.

[12] R. Gao, W. Wu, and J. Liu, "Asian rainbow option pricing formulas of uncertain stock model," Soft Computing, vol. 25, no. 14, pp. 8849-8873, 2021.

[13] X. Wang and L. Wang, "Study on Black-Scholes stock option pricing model based on dynamic investment strategy," International Journal of Innovative Computing, Information and Control, vol. 3, no. 6, pp. 1755-1780, 2007.

[14] X. Wang and L. I. N. Wang, "Study on Black-Scholes option pricing model based on general linear investment strategy (part I: put option)," International Journal of Innovative Computing, Information and Control, vol. 5, no. 6, pp. 1765-1783, 2009.

[15] X. Wang and L. Wang, "Study on Black-Scholes option pricing model based on general linear investment strategy (part II: call option)," International Journal of Innovative Computing, Information and Control, vol. 5, no. 8, pp. 2169-2188, 2009.

[16] M. Li, X. Wang, and F. sun, "Proactive hedging European call option pricing with linear position strategy," Discrete Dynamics in Nature and Society, vol. 2018, Article ID 2087145, 2018.

[17] M. Li, X. Wang, and F. F. Sun, "Pricing of proactive hedging European option with dynamic discrete position strategy," Discrete Dynamics in Nature and Society, vol. 2019, Article ID 1070873, 2019. 
[18] Y. Hu and B. Øksendal, "Fractional white noise calculus and applications to finance," Infinite Dimensional Analysis Quantum Probability and Related Topics, vol. 6, no. 1, pp. 1-32, 2003.

[19] C. Necula, "Option pricing in a fractional Brownian motion environment," Advances in Economic and Financial Research, vol. 2, no. 3, pp. 259-273, 2008. 